\title{
V. Natural nanomaterials
}

Materials are made of atoms. They are called natural materials if they belong to the mineral or living world and if no human modification or processing of their structure has been made. Natural materials have properties at the macroscopic level which are often due to their nano or microstructures.

\section{Nanomaterials in nature}

A natural nanomaterial (figure 39) is a material exhibiting properties which reflect its structure at the nanoscale.

There are plenty of examples of natural nanomaterials; some of them are displayed in figure 40 and discussed in the following sections.

\section{Self-cleaning surfaces}

Lotus flower leaves or taro leaves have a self-cleaning property (figures 41 and 42). Water droplets do not wet the surface. This phenomenon comes from superhydrophobicity, i. e, a very high water repellence. The surface of the lotus leaf is rough on the nanometer scale and covered with a waxy material. This material is hydrophobic and the rough surface reduces the area in contact with the leaf. The combination of both effects makes the droplets of water roll right off, taking out dirt that is on the surface. As a consequence, the surface is self-cleaning.

Nanomaterials which mimic this lotus effect have been developed to create surfaces which remain clean and dry, e. g. self cleaning windows or waterproof clothes.

Contrasting materials, surfaces that capture water also exist in nature. The Namib desert beetle uses special nanostructures to capture moisture from fog. A possible application of this phenomenon could be to manufacture surfaces trapping moisture to use as a water source. 


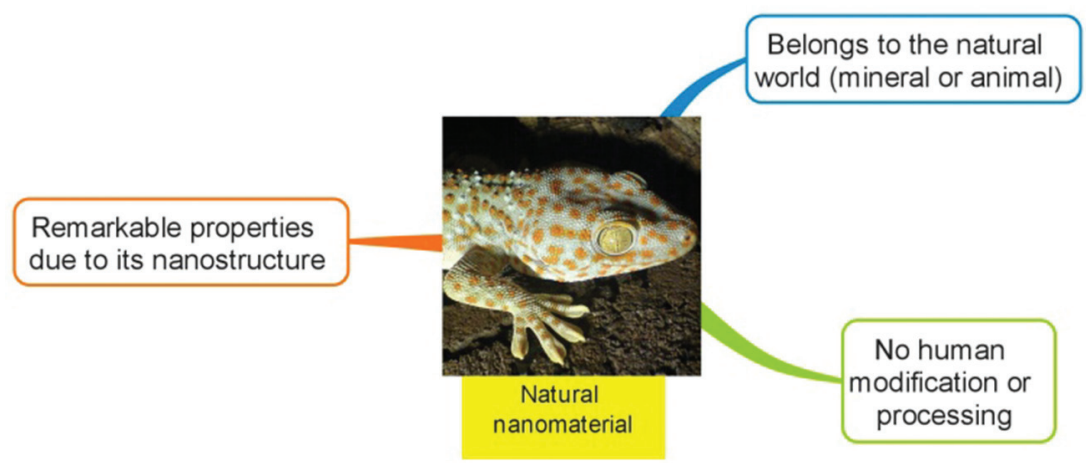

Figure 39. Definition of a natural nanometerial. The image of the Gekko comes from Wikimedia Commons (http://commons.wikimedia.org), author Robert Michniewicz.

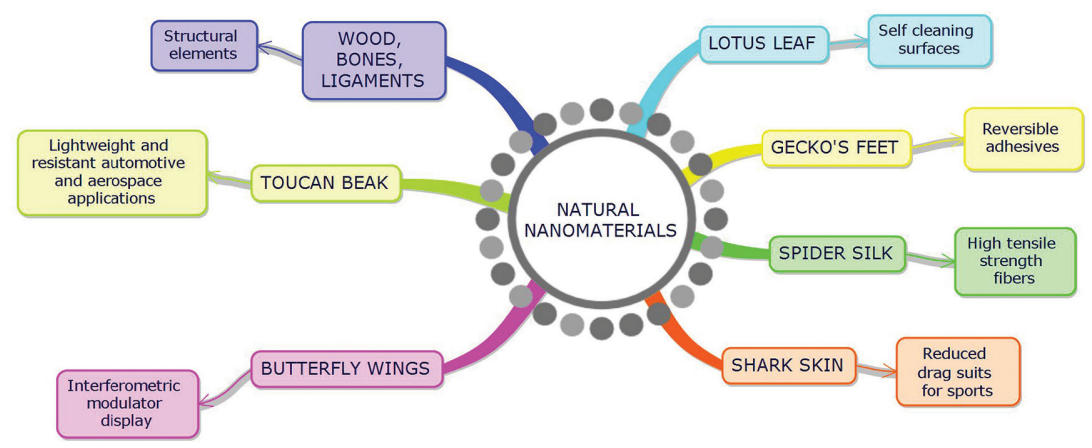

Figure 40. Examples of natural nanomaterials and some biomimetic applications.

\section{Reversible adhesives}

The gecko is a lizard which can cling to any surface at any orientation. It can for example cling and walk on the ceiling with its feet over its head. It can cling on smooth as well as on rough, wet or dirty surfaces. While flies, for example, have similar abilities the gecko is the heaviest animal which can do this.

The weight of an adult Gecko is of the order of $140 \mathrm{~g}$ and, on a vertical wall, it can tolerate about $4 \mathrm{~kg}$ of additional weight without sliding. This astonishing reversible adhesive property is due to millions of setae (tiny bristles and hairs which are typically $100 \mu \mathrm{m}$ long and $5 \mu \mathrm{m}$ in diameter) located on the soles of the gecko's feet. A Gecko foot is shown in figure 43. Each sata ends with hundreds of tiny pads (spatulae, with dimensions of the order of $200 \mathrm{~nm}$ in width and length) helping to cling to a surface (figure 44). Adhesion between the feet and the surface is due to Van der 


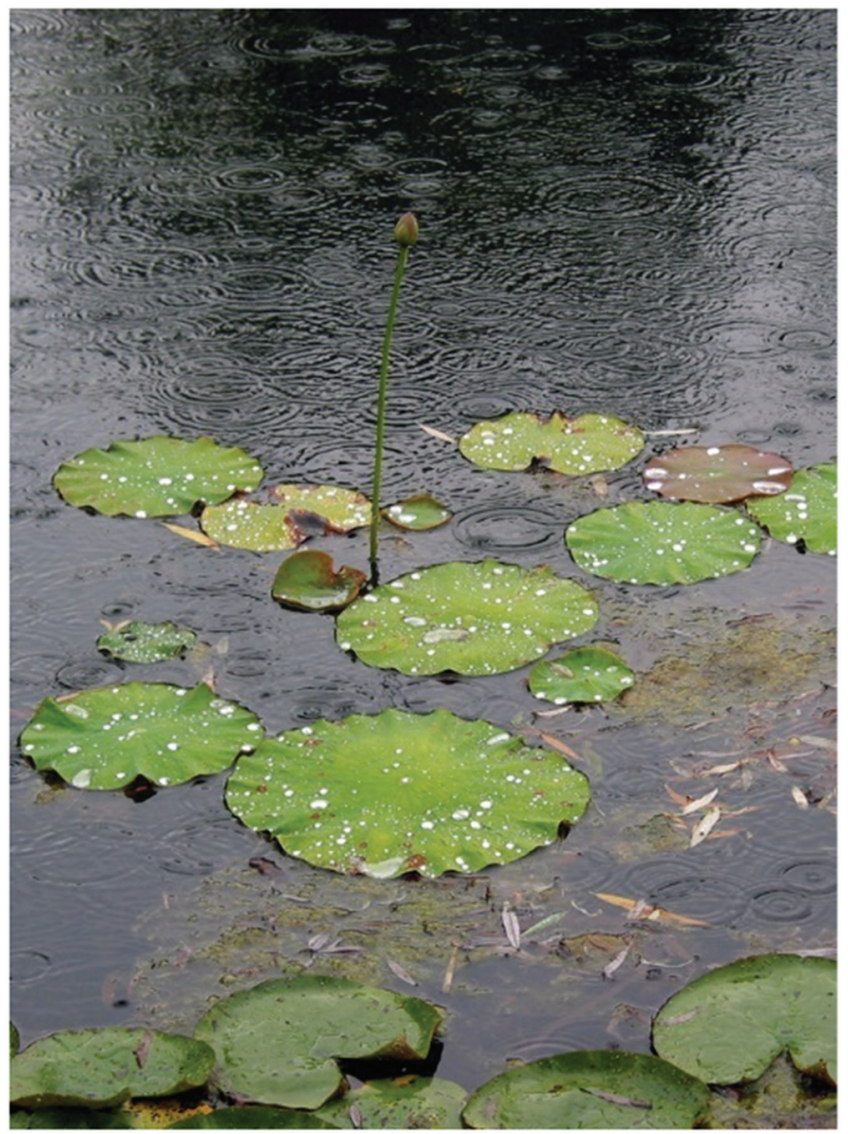

Figure 41. Lotus leaves on pond in rain, showing the so called lotus effect; picture taken in the Chinese Garden at the "Gärten der Welt" (gardens of the world) in Erholungspark Marzahn, Berlin / Germany. Picture from ArchiKat, Wikimedia Commons (http://commons.wikimedia.org).

Waals forces discussed in chapter 3. Since there are a lot of hairs, the total interaction is large and this explains why the adhesion is so strong. The strong adhesive force between the hairs and the surface can be easily relaxed. In other words we have a reversible adhesive. The presence of hairs makes the feet of the Gecko self-cleaning. If dirt-clogged hairs are put on a surface, the dirt particle is left on the surface because there is a smaller contact area between the dirt particle and the satae than between the dirt particle and the surface.

If all the setae on a gecko's feet were active, the gecko could support the weight of two-medium sized persons (a shear force of 1,300 newtons is generated by the $\sim 6.5$ million satae). Actually, the number of active satae 


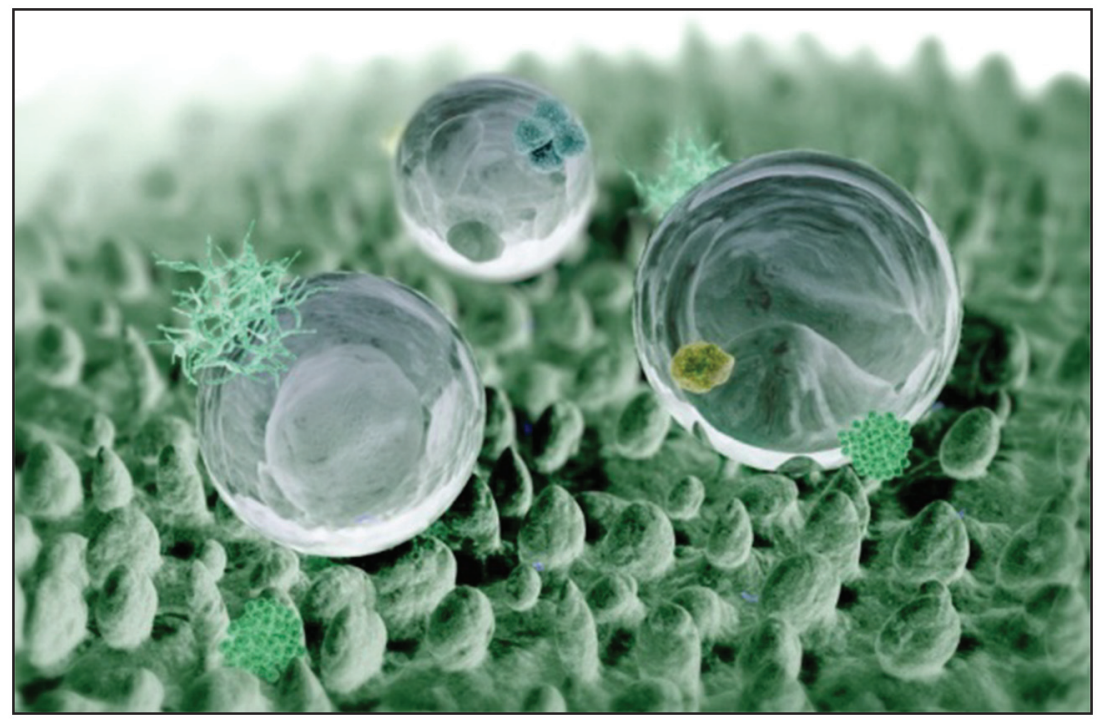

Figure 42. Computer graphics of lotus effect., Picture from Wikimedia Commons (http://commons. wikimedia.org) William Thielicke.

of a gecko is very small, of the order of $3 \%$ but this still creates a very strong force (about 20 newtons).

Several attempts have been made to mimic the Gecko and develop reversible adhesives based on nanoproperties. One goal is surgical band aids that can remain for a long time and which can be removed without pain. The basic idea is to use contact splitting on the nanoscale.This technology was first developed in the US where scientists have used machines usually employed to etch patterns on microchips to create a rubber structure analogous to the gecko's foot. As in the gecko's foot, each hair was covered with thousands of protrusions which are responsible for strong adhesive power. On this nanostructured texture, scientists have added a biodegradable glue giving the ability to the bandage to remain glued when wet. First experiments were made on pigs and rats intestines in 2008 with success. It was found to work better than stitches. For human applications, such band aids can also be impregnated with drugs.

\section{Lightweight materials}

In the aerospace or automotive industries it is important to have strong but light materials. Such materials exist in nature. The beak of a Toucan 


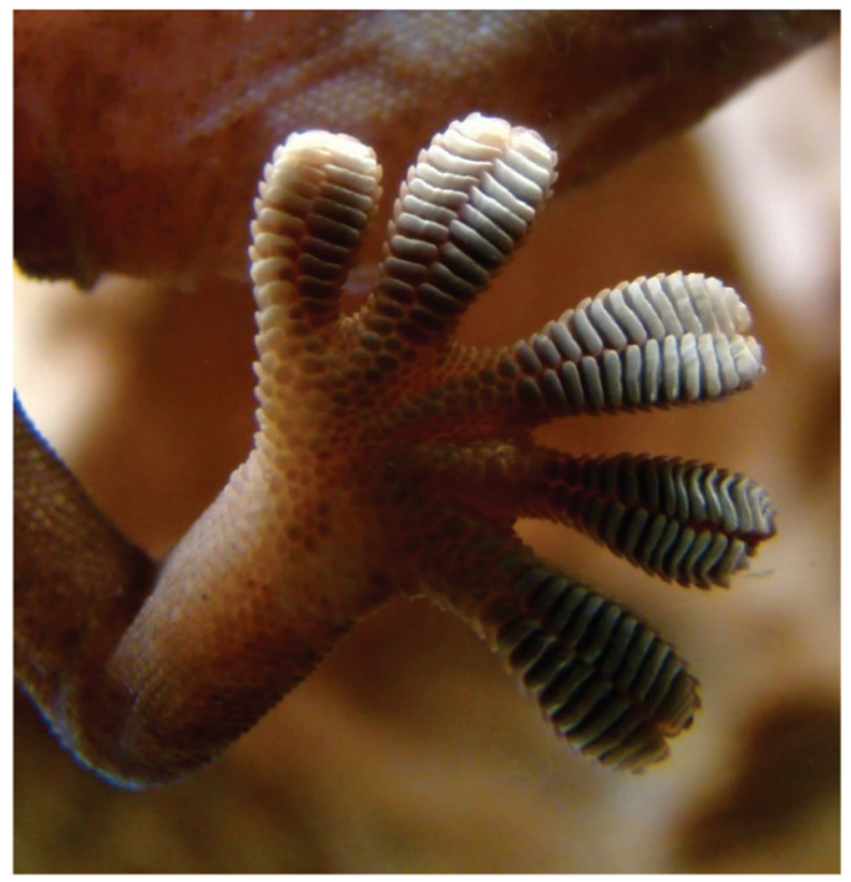

Figure 43. Close-up of the underside of a gecko's foot as it walks on a glass wall. Van der Waals force interactions between the finely divided setae (hairs on the toes) and the glass enables the gecko to stay in place and walk on the seemingly smooth glass. Image from Bjørn Christian Tørrissen, Wikimedia Commons (http://commons.wikimedia.org), and http://bjornfree.com/galleries.html.

(figure 45) is a very strong and light biocomposite. It has hard nanotiles of a protein (keratin which also makes up fingernails, hair and horn) outside and nanosize fibers of bone inside acting as a lightweight supporting foam.

The structure of the toucan beak is interesting because of its high strength to weight ratio and because it is an excellent absorber for high energy impacts. Developing analogous materials for motorist protection in crashes can have many economic and safety implications.

\section{Manipulating light at the nanoscale}

Butterflies (figure 46) manipulate light at the nanoscale thanks to nanometer size structures on their wings. Ridges with dimensions smaller than $1 \mu \mathrm{m}$ have nanostructures allowing for multiple reflection of light leading to beautiful iridescent colors. Similarly constructed artificial structures could be used to develop new fabrics, anti-counterfeit technologies for currency or dye-free paints. 


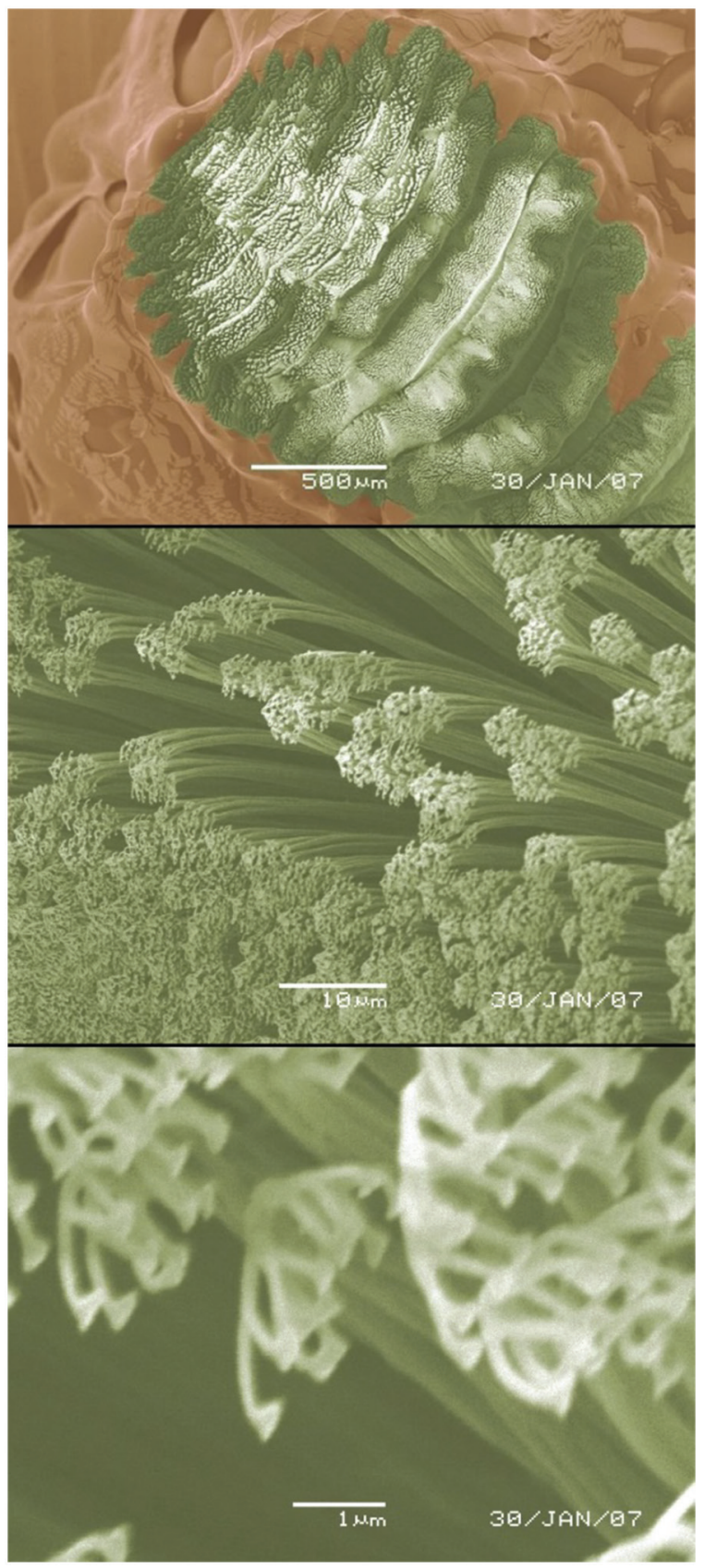

Figure 44. Foot of a Gecko viewed by a scanning electron microscope with increasing magnifications. As magnification increases, we see more and more clearly the tiny hairs. Image from Oskar Gellerbrant, Wikimedia Commons (http://commons.wikimedia.org). 


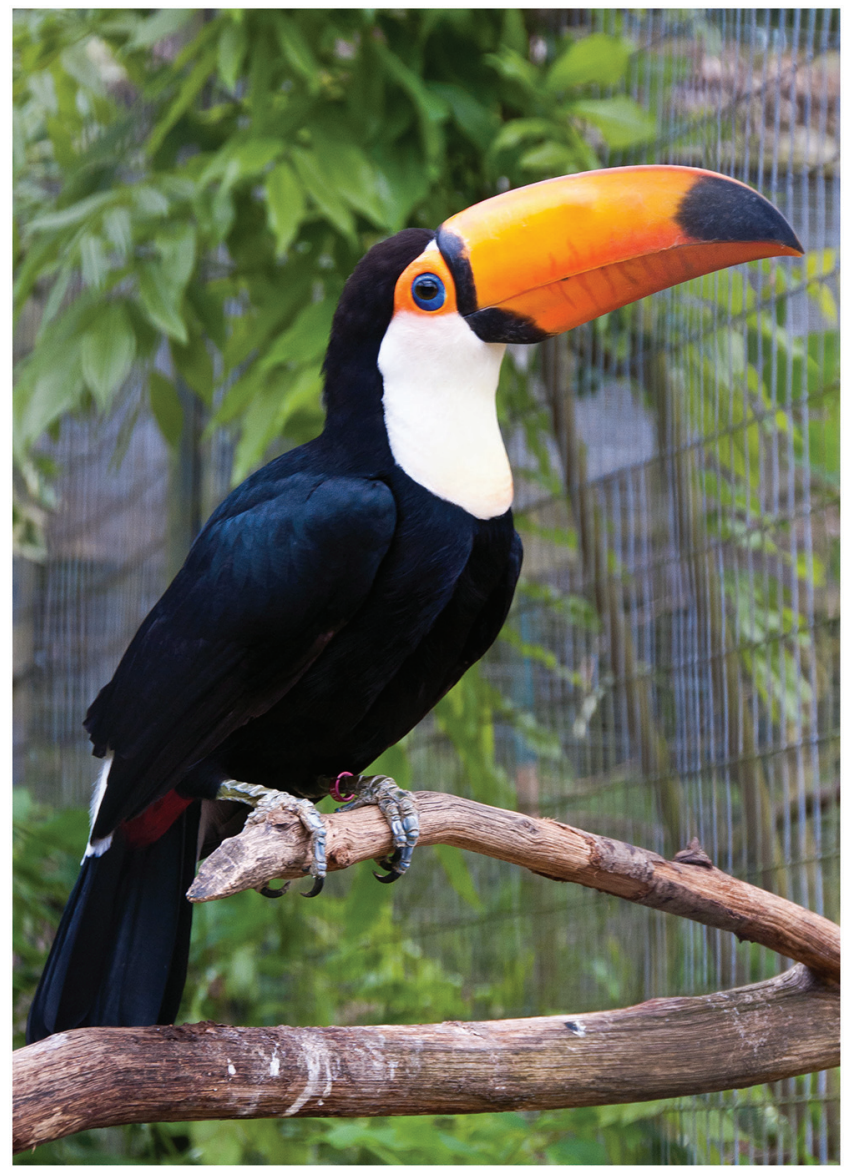

Figure 45. Toucan.

Interferometric modulator displays (IMOD) employ a bio-inspired non -emissive technology where colors are created by controlling how light interferes with itself. The manipulation of light is done with micro-electromechanical systems. One of the advantages is the extremely low energy consumption of displays based on this technology.

\section{Fibers stronger than steel}

The spider web (figure 47) is a very strong natural material. For the same weight, the silk of spider web has a strength about 5 times greater than that of steel. Furthermore, it is elastic and can be stretch up to 10 times its initial length. Spider silk consists of protein molecules produced by 


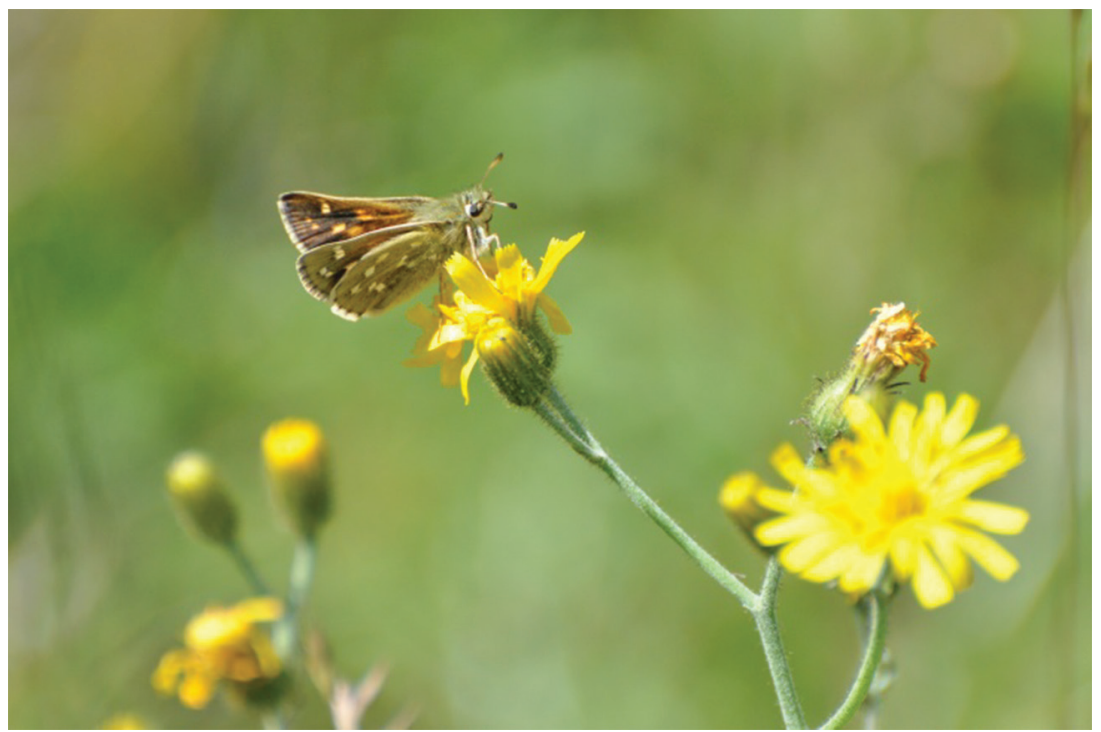

Figure 46. Wings of butterflies have a nanostructure responsible for special optical properties.

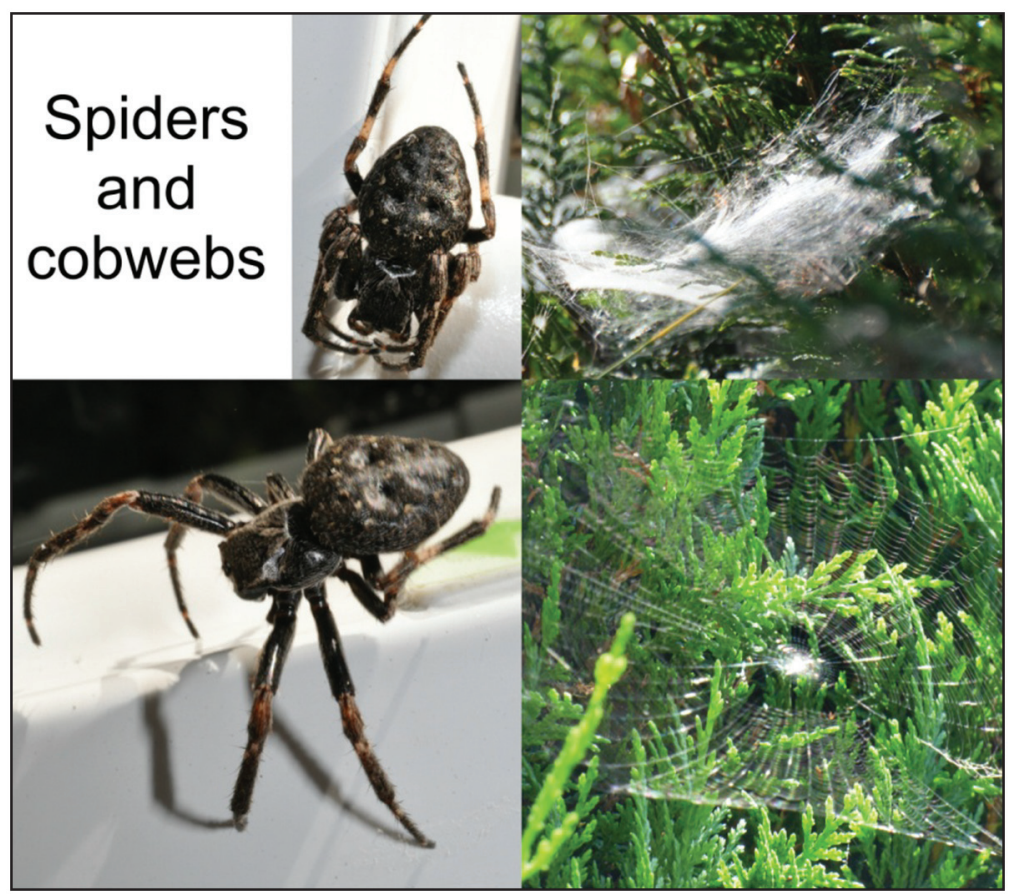

Figure 47. Spider and cobweb. Spider silk is stronger than steel, for the same weight. 
silk glands. The liquid which is produced hardens as it is squeezed outside the body to form silk fibers with outstanding properties. Nanomaterials mirroring the nature of spider silk would have many applications in sport equipment, aeronautics, automobile or household goods as well as in the defense domain, flak vests for example.

\section{Low-friction materials}

The skin of a shark exhibits very low adhesion and large drag reduction for fluid flow. The shark skin is covered with placoid scales (dermal denticles) arranged in a special geometry that reduces friction and turbulent flow during swimming. As a result a shark can reach speeds greater than $70 \mathrm{~km} / \mathrm{h}$.

Several applications have been developed mimicking the shark skin structure. Swimwear has been manufactured to minimize the water resistance. Paints based on nanoparticles have been developed to cover the external surface of boats. The paints are applied using a pattern imitating the structure of the shark skin.These paints also resist UV radiation, temperature and mechanical changes.

Shark skin has also antimicrobial properties and a new material using a a pattern alone derived from shark skin has been recently developed.

\section{Multiscale structures}

Many biological systems are made of self-assembled hierarchical structures over several size scales. This give them outstanding physical properties. This is the case, for example, for bones, wood, skin, teeth and the spider silk we discussed above. At the nanometer scale, there is always a structure which is involved to get excellent mechanical properties. In bones there are multiscale structures but also multiscale responses to deformation. Applying a multiscale philosophy to the development of advanced materials can lead to increased strength and desirable deformation characteristics.

\section{Summary}

Nature is a pool containing very beautiful examples of nanomaterials and nanostructures that lead to unique properties and capabilities. Thoroughly 
investigating these and understanding the underlying structures and the operational mechanisms which are responsible can spur important advances in the development of man-made nanomaterials providing analogous benefits. 